TRANSACTIONS OF THE

AMERICAN MATHEMATICAL SOCIETY

Volume 359, Number 5, May 2007, Pages 2109-2121

S 0002-9947(06)04098-0

Article electronically published on November 22, 2006

\title{
COMPOSITION OPERATORS ON UNIFORM ALGEBRAS, ESSENTIAL NORMS, AND HYPERBOLICALLY BOUNDED SETS
}

\author{
P. GALINDO, T. W. GAMELIN, AND M. LINDSTRÖM
}

\begin{abstract}
Let $A$ be a uniform algebra, and let $\phi$ be a self-map of the spectrum $M_{A}$ of $A$ that induces a composition operator $C_{\phi}$ on $A$. The object of this paper is to relate the notion of "hyperbolic boundedness" introduced by the authors in 2004 to the essential spectrum of $C_{\phi}$. It is shown that the essential spectral radius of $C_{\phi}$ is strictly less than 1 if and only if the image of $M_{A}$ under some iterate $\phi^{n}$ of $\phi$ is hyperbolically bounded. The set of composition operators is partitioned into "hyperbolic vicinities" that are clopen with respect to the essential operator norm. This partition is related to the analogous partition with respect to the uniform operator norm.
\end{abstract}

Some attention has been paid over the years to homomorphisms of uniform algebras, particularly to compact homomorphisms. A unital homomorphism of a uniform algebra $A$ can be realized as a composition operator $C_{\phi}$ given by $f \mapsto f \circ \phi$, where $\phi$ is a self-map of the spectrum $M_{A}$ of $A$. H. Kamowitz [Ka obtained in 1980 a fundamental theorem on compact composition operators asserting the existence of an attracting fixed point for the underlying map $\phi$ of the spectrum. In his pathbreaking 1996 thesis written at Karlsruhe under R. Mortini, U. Klein systematically exploited the contractive properties of the pseudohyperbolic metric on the spectrum to shed light on the Kamowitz theorem and to obtain substantial generalizations. (For an expository account of Klein's thesis, see Ga4.) In order to clarify the natural boundaries of the fixed point theorem along the lines laid out by Klein, we introduced and explored in GGL a notion of "hyperbolic boundedness". In this paper we continue the work in GGL by relating hyperbolic boundedness to the essential spectra of composition operators and to the essential norm of the difference of composition operators.

Our immediate motivation stems from the thesis of L. Zheng [Zh], in which she shows that for an analytic self-map $\phi$ of $\mathbb{D}$, the composition operator $C_{\phi}$ on $H^{\infty}(\mathbb{D})$ has essential spectral radius either 0 or 1 . We extend Zheng's results to the setting of homomorphisms between uniform algebras. P. Gorkin and R. Mortini [GM] have also extended Zheng's work, and our Lemma 2.1 and Corollary 2.2 below are closely related to their Theorems 8 and 9. For related work, see also [HIZ].

Received by the editors February 5, 2004 and, in revised form, February 17, 2005.

2000 Mathematics Subject Classification. Primary 46J10; Secondary 47B38, 47B48.

Key words and phrases. Composition operator, hyperbolically bounded, Gleason part, essential norm.

The first author was supported by Projects AE-2003-0392 (Universidad de Valencia) and BFMFEDER 2003-07540 (DGI, Spain).

The second author was supported partially by the Academy of Finland Project 51096 and Project BFM-FEDER 2003-07540 (DGI, Spain). 
The paper is organized as follows. In Section 2 we give a lower bound for the essential distance between two composition operators, and prove that the essential spectral radius of $C_{\phi}$ is strictly less than 1 if and only if the image of $M_{A}$ under some iterate $\phi^{n}$ of $\phi$ is hyperbolically bounded. In Section 3 we introduce norm vicinities and hyperbolic vicinities of composition operators, and we show that the hyperbolic vicinities partition the set of composition operators. In Section 4 we show that two composition operators belong to the same hyperbolic vicinity if and only if their restrictions to some subalgebra of finite codimension belong to the same norm vicinity. In Section 5 we prove that composition operators whose essential distance is strictly less than 1 belong to the same hyperbolic vicinity and that the hyperbolic vicinities are open (and closed) with respect to the essential operator norm. In Section 6 we determine the hyperbolic vicinity of the identity operator and we show that the identity operator is essentially isolated in the set of composition operators on an algebra whose spectrum has no isolated points. It is also shown that the identity is always isolated in the set of composition operators with the operator norm.

\section{BACKGRound}

Let $A$ be a uniform algebra, with spectrum $M_{A}$. We regard $A$ as an algebra of continuous functions on $M_{A}$, so that $A$ is a closed unital subalgebra of $C\left(M_{A}\right)$. The pseudohyperbolic metric $\rho_{A}(x, y)$ on the spectrum $M_{A}$ is defined by

$$
\rho_{A}(x, y)=\sup \{\rho(f(x), f(y)): f \in A,\|f\|<1\}, \quad x, y \in M_{A},
$$

where $\rho$ is the pseudohyperbolic metric on the open unit disk $\mathbb{D}$ in the complex plane. The pseudohyperbolic metric on $M_{A}$ is expressed in terms of the norm of the evaluation functional at $x$ on the null-space of the evaluation functional at $y$ by

$$
\begin{aligned}
\rho_{A}(x, y) & =\left\|\left.x\right|_{y^{-1}(0)}\right\| \\
& =\sup \{|f(x)|: f \in A,\|f\|<1, f(y)=0\}, \quad x, y \in M_{A} .
\end{aligned}
$$

Evidently $\rho_{A}(x, y) \leq 1$. The pseudohyperbolic metric on $M_{A}$ satisfies König's inequality

$$
\rho_{A}(x, y) \leq \frac{\rho_{A}(x, u)+\rho_{A}(u, y)}{1+\rho_{A}(x, u) \rho_{A}(u, y)}, \quad x, u, y \in M_{A} .
$$

From König's inequality it is easy to see that any two open pseudohyperbolic balls in $M_{A}$ of radius 1 either are disjoint or coincide. These open balls of radius 1 are the Gleason parts of $A$. (See Chapter VI of Ga1.) It is easy to check that

$$
\frac{\|x-y\|}{2} \leq \rho_{A}(x, y) \leq\|x-y\|, \quad x, y \in M_{A}
$$

so that convergence in the pseudohyperbolic metric of $M_{A}$ is tantamount to convergence in the norm of $A^{*}$. The precise relationship between the pseudohyperbolic metric and the norm in $A^{*}$ is given by

$$
\|x-y\|=\frac{2}{\rho_{A}(x, y)}\left(1-\sqrt{1-\rho_{A}(x, y)^{2}}\right), \quad \rho_{A}(x, y)=\frac{4\|x-y\|}{4+\|x-y\|^{2}}
$$

where $x, y \in M_{A}$. (See e.g. [Ko].)

For general background on uniform algebras we refer to Ga1.

The bidual $A^{* *}$ of $A$ is also a uniform algebra. For a description of the bidual of $A$, see [Ga3. The evaluation functionals at points of $M_{A}$ extend uniquely to be 
weak-star continuous multiplicative functionals on $A^{* *}$, so we can regard $M_{A}$ as a subset of the spectrum of $A^{* *}$, and we can regard $A$ as a subalgebra of $A^{* *}$. The restrictions of the functions in $A^{* *}$ to $M_{A}$ are the pointwise limits of bounded nets in $A$.

Following GGL, we define a subset $E$ of $M_{A}$ to be hyperbolically bounded if it is contained in a finite union of pseudohyperbolic balls whose radii are strictly less than 1. Each such ball is contained in a single Gleason part, so that a hyperbolically bounded subset of $M_{A}$ meets only a finite number of Gleason parts of $M_{A}$.

We will make use of the following result, which is Theorem 2.5 in GGL.

Theorem 1.1. Let $A$ be a uniform algebra, with spectrum $M_{A}$ and bidual $A^{* *}$. Let $E$ be a subset of $M_{A}$ that is not hyperbolically bounded. Then for each $\varepsilon>0$, there are a sequence of points $\left\{x_{j}\right\}_{j=1}^{\infty}$ in $E$ and a sequence of functions $\left\{F_{k}\right\}_{k=1}^{\infty}$ in $A^{* *}$ such that $F_{k}\left(x_{j}\right)=0$ for $j \neq k, F_{k}\left(x_{k}\right)=1$, and $\sum_{k=1}^{\infty}\left|F_{k}\right| \leq 1+\varepsilon$ on $M_{A^{* *}}$.

Note that the sequence $\left\{F_{k}\right\}$ in the theorem converges weakly in $A^{* *}$ to 0 . In fact, for a sequence of functions $\left\{f_{k}\right\}$ in a subspace of $C(X)$, the condition $\sum\left|f_{k}(x)\right| \leq$ $M<\infty$ holds if and only if the correspondence $e_{k} \mapsto f_{k}$ extends to a continuous linear operator from the sequence space $c_{0}$ into the subspace. (See e.g. Da.) Since the standard basis $\left\{e_{k}\right\}$ for $c_{0}$ tends weakly to 0 , so does $\left\{f_{k}\right\}$. (This can also be seen by representing a continuous linear functional on $C(X)$ by a measure and applying the Lebesgue dominated convergence theorem.)

A composition operator from a uniform algebra $A$ to a uniform algebra $B$ is an operator of the form $C_{\phi}: f \mapsto f \circ \phi$, where $\phi: M_{B} \rightarrow M_{A}$ is continuous. The composition operators from $A$ to $B$ are precisely the unital homomorphisms from $A$ to $B$. Composition operators satisfy $\left\|C_{\phi}\right\|=1=C_{\phi}(1)$. If $C_{\phi}$ is a composition operator from $A$ to $A$, then $C_{\phi}^{n}=C_{\phi^{n}}$, where $\phi^{n}=\phi \circ \cdots \circ \phi$ is the $n$th iterate of $\phi$.

\section{ESSENTIAL SPECTRAL RADIUS OF COMPOSITION OPERATORS}

We denote the essential norm of an operator $T$ from $A$ to $B$ by $\|T\|_{e}$. The essential norm is the distance from $T$ to the compact operators from $A$ to $B$,

$$
\|T\|_{e}=\inf \{\|T+K\|: K \text { a compact operator }\}
$$

Theorem 1.1 leads to a brief proof of the following lemma. (See Theorems 8 and 9 of P. Gorkin and R. Mortini GM]. See also [HIZ.)

Lemma 2.1. Let $C_{\phi}$ and $C_{\psi}$ be composition operators from $A$ to $B$. If $\left\{y_{j}\right\}$ is a sequence in $M_{B}$ such that $\left\{\phi\left(y_{j}\right)\right\}$ is not hyperbolically bounded in $M_{A}$, then

$$
\left\|C_{\phi}-C_{\psi}\right\|_{e} \geq \liminf \rho_{A}\left(\phi\left(y_{j}\right), \psi\left(y_{j}\right)\right) .
$$

Proof. Set $\lim \inf \rho_{A}\left(\phi\left(y_{j}\right), \psi\left(y_{j}\right)\right)=L$. Let $K$ be a compact operator from $A$ to $B$, and let $\varepsilon>0$. Passing to a subsequence and applying Theorem 1.1 to $\left\{\phi\left(y_{j}\right)\right\}$, we can find a sequence of functions $\left\{F_{k}\right\}_{k=1}^{\infty}$ in $A^{* *}$ such that $F_{k}\left(\phi\left(y_{j}\right)\right)=0$ for $j \neq k$, $F_{k}\left(\phi\left(y_{k}\right)\right)=1$, and $\sum_{k=1}^{\infty}\left|F_{k}\right| \leq 1+\varepsilon$ on $M_{A^{* *}}$. Take $g_{k} \in A$ such that $\left\|g_{k}\right\|<1$, $g_{k}\left(\psi\left(y_{k}\right)\right)=0$, and $g_{k}\left(\phi\left(y_{k}\right)\right) \rightarrow L$. Since $\sum\left|g_{k} F_{k}\right| \leq 1+\varepsilon, g_{k} F_{k} \rightarrow 0$ weakly in 
$A^{* *}$. As $K^{* *}$ is also compact, $\left\|K^{* *}\left(g_{k} F_{k}\right)\right\| \rightarrow 0$. Consequently we obtain

$$
\begin{aligned}
& (1+\varepsilon)\left\|C_{\phi}-C_{\psi}+K\right\| \geq \limsup \left\|\left(C_{\phi}^{* *}-C_{\psi}^{* *}+K^{* *}\right)\left(g_{k} F_{k}\right)\right\| \\
& \quad=\limsup \left\|\left(C_{\phi}^{* *}-C_{\psi}^{* *}\right)\left(g_{k} F_{k}\right)\right\| \\
& \quad \geq \limsup \left|\left(C_{\phi}^{* *}\left(g_{k} F_{k}\right)\right)\left(y_{k}\right)-\left(C_{\psi}^{* *}\left(g_{k} F_{k}\right)\right)\left(y_{k}\right)\right| \\
& \quad=\limsup \left|\left(g_{k} F_{k}\right)\left(\phi\left(y_{k}\right)\right)-\left(g_{k} F_{k}\right)\left(\psi\left(y_{k}\right)\right)\right|=\lim \left|g_{k}\left(\phi\left(y_{k}\right)\right)\right|=L .
\end{aligned}
$$

It follows that $\left\|C_{\phi}-C_{\psi}\right\|_{e} \geq L$, as required.

Corollary 2.2. Let $C_{\phi}$ be a composition operator from a uniform algebra $A$ to a uniform algebra $B$. If $\phi\left(M_{B}\right)$ is not a hyperbolically bounded subset of $M_{A}$, then $\left\|C_{\phi}\right\|_{e}=1$.

Proof. Since $\left\|C_{\phi}\right\| \leq 1$, also $\left\|C_{\phi}\right\|_{e} \leq 1$. For the reverse inequality, take $\psi$ as any constant mapping with value, say, $a \in M_{A}$. Since $\phi\left(M_{B}\right)$ is not a hyperbolically bounded, there is sequence $\left\{\phi\left(y_{j}\right)\right\}$ such that $\lim \rho_{A}\left(\phi\left(y_{j}\right), a\right)=1$. Clearly, $C_{\psi}$ is a finite rank operator, so $\left\|C_{\phi}\right\|_{e}=\left\|C_{\phi}-C_{\psi}\right\|_{e} \geq 1$ by the lemma above.

We denote the essential spectrum of an operator $T$ on $A$ by $\sigma_{e}(T)$. Thus $\sigma_{e}(T)$ is the spectrum of the coset of $T$ in the quotient Banach algebra of operators on $A$ modulo compact operators. It is a compact subset of the complex plane, which is nonempty except in the trivial case that $A$ is finite dimensional. We denote by $r_{e}(T)$ the essential spectral radius of $T$, that is, the supremum of $|\lambda|$ for $\lambda \in \sigma_{e}(T)$. By the spectral radius formula, $r_{e}(T)=\lim \left\|T^{n}\right\|_{e}^{1 / n}$.

Corollary 2.2 allows us to expand upon Theorem 3.3 of GGL.

Theorem 2.3. Let $C_{\phi}$ be a unital homomorphism of the uniform algebra $A$. The following statements are equivalent:

(i) There is a decomposition of $M_{A}$ into disjoint clopen subsets $E_{1}, \ldots, E_{m}$ such that the iterates of $\phi$ converge uniformly on each $E_{j}$ in the pseudohyperbolic metric to an attracting cycle in $E_{j}$ for $\phi$.

(ii) There is $n \geq 1$ such that the $n$th iterate $\phi^{n}\left(M_{A}\right)$ of $M_{A}$ under $\phi$ is a hyperbolically bounded subset of $M_{A}$.

(iii) $r_{e}\left(C_{\phi}\right)<1$, that is, the essential spectrum of $C_{\phi}$ does not contain any points of the unit circle.

Proof. The equivalence of (i) and (ii) is established in Theorem 3.3 of GGL. If (ii) fails, then the preceding lemma shows that $\left\|C_{\phi}^{n}\right\|_{e}=1$ for all $n \geq 1$. By the spectral radius formula, $r_{e}\left(C_{\phi}\right)=1$, and (iii) also fails. Thus (iii) implies (ii).

Suppose that (i) and (ii) hold. Replacing $\phi$ by some iterate $\phi^{N}$, we can assume that the attracting cycles in (i) are attracting fixed points, that $\phi\left(M_{A}\right)$ is hyperbolically bounded and, as easily seen from the proof Theorem 3.3 in GGL, that the sets $E_{j}$ are invariant under $\phi$. By looking at the inverse image in $E_{j}$ of pseudohyperbolic balls of radius less than 1 , we may also assume that $\phi\left(E_{j}\right)$ lies in such a single ball of radius $r<1$. Note that by König's inequality this ball has $\rho_{A^{-}}$ diameter less than $\frac{2 r}{1+r^{2}}$. Then $\phi$ is a pseudohyperbolic contraction with contraction constant $c=\frac{2 r}{1+r^{2}}<1$ on each of the sets $\phi\left(E_{j}\right)$. Indeed: For $x, y \in \phi\left(E_{j}\right)$ and $f \in A,\|f\|<1$ with $f(\phi(y))=0$, the function $f \chi_{E_{j}} \circ \phi \in A$ and $\left\|f \chi_{E_{j}} \circ \phi\right\| \leq c$, and therefore, $\left|\frac{1}{c} f \chi_{E_{j}} \circ \phi(x)\right| \leq \rho_{A}(x, y)$. Thus $|f(\phi(x))| \leq c \rho_{A}(x, y)$, and hence $\rho_{A}(\phi(x), \phi(y)) \leq c \rho_{A}(x, y)$. 
Let $A_{0}$ be the subspace of functions in $A$ that vanish at the fixed points $\left\{x_{1}, \ldots\right.$, $\left.x_{m}\right\}$. Then $A_{0}$ is an invariant subspace for $C_{\phi}$. If $f \in A_{0},\|f\|<1, x \in E_{j}$, and $y=\phi(x)$, then $\left|\left(C_{\phi}^{k+1} f\right)(x)\right|=\left|f\left(\phi^{k+1}(x)\right)\right|=\left|f\left(\phi^{k}(y)\right)\right| \leq \rho_{A}\left(\phi^{k}(y), x_{j}\right) \leq$ $c^{k} \rho_{A}\left(y, x_{j}\right) \leq c^{k}$. Thus the norm of the restriction of $C_{\phi}^{k+1}$ to $A_{0}$ is bounded by $c^{k}$. Taking $(k+1)$ th roots and sending $k$ to $\infty$, we find that the spectral radius of the restriction of $C_{\phi}$ to $A_{0}$ is at most $c$. Since $A_{0}$ has finite codimension in $A$, the essential spectral radius of $C_{\phi}$ is at most $c$, and (iii) holds.

A subset $E$ of the spectrum $M_{B}$ of a uniform algebra $B$ is a norming set if

$$
\|f\|=\sup \{|f(x)|: x \in E\}, \quad f \in B .
$$

According to Lemma 3.8 of GGL, if $\phi: M_{B} \rightarrow M_{A}$ corresponds to a composition operator $C_{\phi}: A \rightarrow B$, then $\phi\left(M_{B}\right)$ is hyperbolically bounded in $M_{A}$ as soon as $\phi(E)$ is hyperbolically bounded in $M_{A}$. This leads to the following corollary.

Corollary 2.4. Let $B_{X}$ be the open unit ball of a Banach space $X$, and let $A$ be a uniformly closed subalgebra of $H^{\infty}\left(B_{X}\right)$ that contains the functions in $X^{*}$. Let $\phi: B_{X} \mapsto B_{X}$ be an analytic map that induces a composition operator on $A$. Then $r_{e}\left(C_{\phi}\right)<1$ if and only if there are $r<1$ and an integer $n \geq 1$ such that $\phi^{n}\left(B_{X}\right) \subset r B_{X}$.

Proof. Since $B_{X}$ is a norming set for $A$, Lemma 3.8 of [GGL] shows that $\phi^{n}\left(M_{A}\right)$ is hyperbolically bounded if and only if $\phi^{n}\left(B_{X}\right)$ is hyperbolically bounded. Since $\rho_{A}(0, x)=\|x\|$ for $x \in B_{X}$, this occurs if and only if $\phi^{n}\left(B_{X}\right)$ is contained in a ball $r B_{X}$ for some $r<1$. Thus the corollary follows from the equivalence of (ii) and (iii) in Theorem 2.3.

If the Banach space $X$ is finite dimensional, then either $r_{e}\left(C_{\phi}\right)=1$ or $r_{e}\left(C_{\phi}\right)=0$. Indeed, if $r_{e}\left(C_{\phi}\right)<1$, then for $n$ large, $\phi^{n}\left(B_{X}\right) \subseteq r B_{X}$, and consequently $C_{\phi}^{n}$ is a compact operator. It follows that $r_{e}\left(C_{\phi}\right)=0$ and $\sigma_{e}\left(C_{\phi}\right)=\{0\}$. The same conclusion holds if $A$ is a logmodular algebra, or more generally a URM-algebra (an algebra for which every $x \in M_{A}$ has a unique representing measure on the Shilov boundary; see GL).

If the Banach space $X$ is infinite dimensional, it can occur that $0<r_{e}\left(C_{\phi}\right)<1$. Indeed, let $\lambda$ be a complex number, $0<|\lambda|<1$, and define $\phi(x)=\lambda x$ for $x \in B_{X}$. The space $\mathcal{P}^{k}$ of homogeneous polynomials on $X$ of degree $k$ is an eigenspace for $C_{\phi}$ with eigenvalue $\lambda^{k}$. The eigenspace $\mathcal{P}^{0}$ is one dimensional, consisting only of the constant functions, while $\mathcal{P}^{1}=X^{*}$. Since the eigenspaces $\mathcal{P}^{k}$ are infinite dimensional for $k \geq 1$, the essential spectrum of $C_{\phi}$ includes $\lambda^{k}$ for $k \geq 1$. Let us see that $\sigma\left(C_{\phi}\right)=\left\{0,1, \lambda, \lambda^{2}, \ldots\right\}$. First, note that $\left\{\lambda^{k}: k=0,1, \ldots\right\}$ are the only eigenvalues of $C_{\phi}$. For $\mu \notin\left\{0,1, \lambda, \lambda^{2}, \ldots\right\}$ choose $n \in \mathbb{N}$ such that $|\lambda|^{n}<|\mu|$ and let us check that the one-to-one map $\mu-C_{\phi}$ is an onto map. Consider the natural direct sum decomposition

$$
H^{\infty}\left(B_{X}\right)=\mathcal{P}^{0} \oplus \mathcal{P}^{1} \oplus \cdots \oplus \mathcal{P}^{n} \oplus \mathcal{Q},
$$

where $|f(x)| \leq\|f\|\|x\|^{n+1}$ for $f \in \mathcal{Q}$ and $x \in B_{X}$. For any $f \in \mathcal{Q}$, the series $g:=\mu^{-1} \sum_{0}^{\infty}\left(f \circ \phi^{k}\right) \mu^{-k}$ is uniformly convergent in $H^{\infty}\left(B_{X}\right)$ and $\left(\mu-C_{\phi}\right)(g)=f$. Moreover the subspace $\mathcal{P}^{0} \oplus \mathcal{P}^{1} \oplus \cdots \oplus \mathcal{P}^{n}$ is contained in the range of the map $\mu-C_{\phi}$ which thus turns out to be an onto mapping. Since $1 \notin \sigma_{e}\left(C_{\phi}\right)$, we further obtain $\sigma_{e}\left(C_{\phi}\right)=\sigma\left(C_{\phi}\right) \backslash\{1\}=\left\{0, \lambda, \lambda^{2}, \ldots\right\}$ and $r_{e}\left(C_{\phi}\right)=|\lambda|$. 
Consider now the one-dimensional case of the open unit disk $\mathbb{D}$ and a composition operator $C_{\phi}$ on $H^{\infty}(\mathbb{D})$ arising from an analytic map $\phi: \mathbb{D} \rightarrow \mathbb{D}$. As mentioned before, Zheng [Zh] proved that either $r_{e}\left(C_{\phi}\right)=0$ or $r_{e}\left(C_{\phi}\right)=1$, depending on whether there is $n \geq 1$ such that $\phi^{n}(\mathbb{D})$ is a relatively compact subset of $\mathbb{D}$. Further, Zheng showed that if $r_{e}\left(C_{\phi}\right)=1$, and if $\phi$ has an attracting fixed point in $\mathbb{D}$, then the spectrum of $C_{\phi}$ fills out the closed unit disk $\overline{\mathbb{D}}$. It would be of interest to determine in a general setting exactly when $\sigma\left(C_{\phi}\right)=\overline{\mathbb{D}}$.

\section{Hyperbolic Vicinities of COMPOSITION OPERATORS}

We consider two composition operators $C_{\phi}$ and $C_{\psi}$ from the uniform algebra $A$ to a uniform algebra $B$. In analogy with the usual definition of Gleason parts, we say that $C_{\phi}$ and $C_{\psi}$ are in the same norm vicinity if $\left\|C_{\phi}-C_{\psi}\right\|<2$, that is, if

$$
\sup _{f \in \text { ball } A, y \in M_{B}}|f(\phi(y))-f(\psi(y))|=\sup _{y \in M_{B}}\|\phi(y)-\psi(y)\|<2 .
$$

In view of the relation (4) between the norm and the pseudohyperbolic metric, this occurs if and only if there is $r<1$ such that

$$
\rho_{A}(\phi(y), \psi(y)) \leq r, \quad y \in M_{B}
$$

As in the case of Gleason parts, being in the same vicinity is an equivalence relation. The norm vicinities form a partition of the set of composition operators from $A$ to $B$ into pairwise disjoint sets that are clopen with respect to the uniform operator norm.

We would like to develop a notion of vicinity that is adapted to the essential operator norm rather than the uniform operator norm. Towards this goal, we make the following definition.

Definition. Composition operators $C_{\phi}$ and $C_{\psi}$ from $A$ to $B$ are in the same hyperbolic vicinity if there are a norming set $Y$ for $B$, a subset $E$ of $Y$, and an $r<1$ such that

(i) $\rho_{A}(\phi(y), \psi(y)) \leq r$ for all $y \in E$, and

(ii) $\phi(Y \backslash E)$ and $\psi(Y \backslash E)$ are hyperbolically bounded in $M_{A}$.

Each norm vicinity is contained in a hyperbolic vicinity. We aim to eventually show that the hyperbolic vicinities partition the set of composition operators from $A$ to $B$ into pairwise disjoint sets that are clopen with respect to the essential operator norm. We begin with some lemmas.

Lemma 3.1. Let $x_{0} \in M_{A}$ and let $r<1$. The pseudohyperbolic closed ball $B_{r}=$ $\left\{x \in M_{A}: \rho_{A}\left(x, x_{0}\right) \leq r\right\}$ is a closed $A$-convex subset of $M_{A}$. Further, if $f_{n} \in A$ satisfy $\left\|f_{n}\right\|<1$ and $f_{n}\left(x_{0}\right) \rightarrow 1$, then $f_{n} \rightarrow 1$ uniformly on $B_{r}$.

Proof. By (2), $B_{r}$ is the set of $x \in M_{A}$ such that $|f(x)| \leq r$ for all $f \in A$ satisfying $\|f\|<1$ and $f\left(x_{0}\right)=0$. The first statement follows immediately from this description. The second statement follows from the definition (1) of $\rho_{A}$ and the fact that if the centers of pseudohyperbolic disks of radius $r$ in $\mathbb{D}$ tend to 1 , then the disks tend to 1 .

Lemma 3.2. If the subset $E$ of $M_{A}$ is hyperbolically bounded, then the A-convex hull $\widehat{E}$ of $E$ is hyperbolically bounded. Further, if $P_{1}, \ldots, P_{m}$ are distinct Gleason parts, and if $E=\bigcup_{j=1}^{m} E_{j}$ where $E_{j}$ is a hyperbolically bounded subset of $P_{j}$, then $\widehat{E_{j}} \subset P_{j}$, and $\widehat{E}=\bigcup_{j=1}^{m} \widehat{E_{j}}$. 
Proof. Suppose $E$ is contained in a union of closed pseudohyperbolic balls of the form $B_{j}=\left\{\rho_{A}\left(x, x_{j}\right) \leq r_{j}\right\}$, where $x_{j} \in P_{j}$. By Lemma 3.1, $\widehat{E_{j}} \subset B_{j}$. Since $x_{1}$ and $x_{2}$ are in different Gleason parts, there are functions $f_{n} \in A$ satisfying $\left\|f_{n}\right\|<1$, $f_{n}\left(x_{1}\right) \rightarrow 1$, and $f_{n}\left(x_{2}\right) \rightarrow-1$. By Lemma $3.1, f_{n} \rightarrow 1$ uniformly on $B_{1}$, and also $f_{n} \rightarrow-1$ uniformly on $B_{2}$. Thus $\left(1+f_{n}\right) / 2$ converges uniformly to 1 on $B_{1}$ and to 0 on $B_{2}$. By taking products of such functions constructed for the various $x_{j}$ 's, we see that the function that is 1 on $B_{k}$ and 0 on the other $B_{j}$ 's can be approximated uniformly on $\bigcup B_{j}$ by functions in $A$. Thus the spectrum $\widehat{E}=\widehat{\bigcup E_{j}}$ of the uniform closure of the restriction of $A$ to $\bigcup E_{j}$ coincides with $\bigcup \widehat{E_{j}}$.

Lemma 3.3. Let $C_{\phi}$ and $C_{\psi}$ be composition operators from $A$ to $B$, and let $E$ be a subset of $M_{B}$. If $\rho_{A}(\phi(y), \psi(y)) \leq r$ for $y \in E$, then $\rho_{A}(\phi(y), \psi(y)) \leq r$ for $y \in \widehat{E}$.

Proof. In view of the relation (4) between the norm and the pseudohyperbolic metric, it suffices to show that if $\|\phi(y)-\psi(y)\| \leq c$ for $y \in E$, then $\|\phi(y)-\psi(y)\| \leq c$ for $y \in \widehat{E}$. So suppose that $\|\phi(y)-\psi(y)\| \leq c$ for $y \in E$. Then $|f(\phi(y))-f(\psi(y))|=$ $\left|\left(C_{\phi} f\right)(y)-\left(C_{\psi} f\right)(y)\right| \leq c$ for $f \in$ ball $A$ and $y \in E$. For each $f$, this estimate persists for $y \in \widehat{E}$. Consequently $\|\phi(y)-\psi(y)\| \leq c$ for $y \in \widehat{E}$.

Theorem 3.4. Let $C_{\phi}$ and $C_{\psi}$ be composition operators from $A$ to $B$ that belong to the same hyperbolic vicinity, and let $E$ be the set of $y \in M_{B}$ such that $\phi(y)$ and $\psi(y)$ belong to the same Gleason part of $M_{A}$. Then $E$ is a clopen subset of $M_{B}$, and there is $r<1$ such that $\rho_{A}(\phi(y), \psi(y)) \leq r$ for $y \in E$. Further, $M_{B}$ is the disjoint union of $E$ and a finite number of disjoint clopen sets $E_{\alpha}$ such that for each index $\alpha, \phi\left(E_{\alpha}\right)$ and $\psi\left(E_{\alpha}\right)$ are hyperbolically bounded subsets of different Gleason parts.

Proof. Let $Y$ and $E$ be as in the definition of hyperbolic vicinity. Adjusting $r$ if necessary, we can adjoin to $E$ any subset $S$ of $M_{B}$ such that $\phi(S)$ and $\psi(S)$ are hyperbolically bounded subsets of the same part. Making a finite number of such adjunctions and adjustments, we can assume that $E$ consists of all $y \in Y$ such that $\phi(y)$ and $\psi(y)$ belong to the same part.

Now $\phi(Y \backslash E) \cup \psi(Y \backslash E)$ is contained in a finite union of Gleason parts $P_{1}, \ldots, P_{m}$ of $M_{A}$. For $j \neq k$, let $E_{j k}$ be the set of $y \in Y$ such that $\phi(y) \in P_{j}$ and $\psi(y) \in P_{k}$. (Some of the $E_{j k}$ 's may be empty.) Then $Y$ is the disjoint union of $E$ and the $E_{j k}$ 's. By Lemma $3.2, \phi\left(\widehat{E_{j k}}\right)$ and $\psi\left(\widehat{E_{j k}}\right)$ are hyperbolically bounded subsets of $P_{j}$ and $P_{k}$, respectively. By Lemma 3.3, $\rho_{A}(\phi(y), \psi(y)) \leq r$ for $y \in \widehat{E}$. Thus $\widehat{E}$ and the $\widehat{E_{j k}}$ 's are disjoint subsets of $M_{B}$.

Let $F(z, w)=(1+z)(1-w) / 4$. Then $F(1,-1)=1$, and $|F(z, w)|<1$ for $|z| \leq 1$, $|w| \leq 1,(z, w) \neq(1,-1)$. There is a constant $c=c(r)<1$ such that if $\zeta, \xi \in \mathbb{D}$ satisfy $\rho(\zeta, \xi) \leq r$, then $|F(\zeta, \xi)| \leq c$. Thus if $f \in A$ satisfies $\|f\|<1$, and if $y \in E$, then $|F(f(\phi(y)), f(\psi(y)))| \leq c$. For any two pseudohyperbolic balls of radii $<1$ in different Gleason parts, we can find a sequence of functions in the open unit ball of $A$ that converges uniformly to 1 on one of the pseudohyperbolic balls and to -1 (or to 0 ) on the other. By combining such sequences, as in the proof of Lemma 3.2, we can find a sequence of functions $\left\{f_{n}\right\}$ in $A$ such that $\left\|f_{n}\right\|<1, f_{n} \rightarrow 1$ uniformly on $\phi\left(E_{12}\right) \subset P_{1}, f_{n} \rightarrow-1$ uniformly on $\psi\left(E_{12}\right) \subset P_{2}$, and $f_{n} \rightarrow 0$ uniformly on subballs of $P_{j}$ for $j \geq 3$. Set $g_{n}=F\left(f_{n} \circ \phi, f_{n} \circ \psi\right) \in B$. Then $\left\|g_{n}\right\|<1, g_{n} \rightarrow 1$ uniformly on $E_{12}, g_{n} \rightarrow 0$ uniformly on $E_{21}, g_{n} \rightarrow 1 / 4$ uniformly on the other $E_{j k}$ 's, and $\left|g_{n}\right| \leq c$ on $E$. If $\left\{k_{n}\right\}$ is a sequence of integers that tends very slowly 
to $+\infty$, then $g_{n}^{k_{n}}$ still converges to 1 uniformly on $E_{12}$, while $g_{n}^{k_{n}} \rightarrow 0$ uniformly on the other $E_{j k}$ 's and on $E$. In this way we see that the characteristic function of each $E_{j k}$, regarded as a function on $Y$, belongs to $B$. Since $Y$ is a norming set for $B, M_{B}$ is then the disjoint union of $\widehat{E}$ and the $\widehat{E_{j k}}$ 's.

The relation of belonging to the same hyperbolic vicinity is clearly reflexive and symmetric. This theorem shows that we may take as a norming set in the definition of hyperbolic vicinity the whole spectrum $M_{B}$ and therefore, transitivity follows easily from it and König's inequality (3).

If $M_{B}$ is connected, then only one of the sets among $E$ and the $E_{\alpha}$ 's can be nonempty, and we obtain the following.

Corollary 3.5. Let $C_{\phi}$ and $C_{\psi}$ be composition operators from $A$ to $B$ that belong to the same hyperbolic vicinity. Suppose that $M_{B}$ is connected. Then either $C_{\phi}$ and $C_{\psi}$ belong to the same norm vicinity, or $\phi\left(M_{B}\right)$ and $\psi\left(M_{B}\right)$ are hyperbolically bounded subsets of two different Gleason parts of $M_{A}$.

Thus if $M_{B}$ is connected, each hyperbolic vicinity is a norm vicinity, with one exception. The exception is the grand hyperbolic vicinity consisting of all composition operators $C_{\phi}$ from $A$ to $B$ such that $\phi\left(M_{B}\right)$ is hyperbolically bounded. If $B=H^{\infty}(\mathbb{D})$, or more generally if $B$ is a URM-algebra with connected spectrum, then this grand hyperbolic vicinity coincides with the set of compact composition operators on $B$.

Corollary 3.6. Suppose $\phi$ and $\psi$ are analytic self-maps of some connected analytic set $V$. Then the induced composition operators $C_{\phi}$ and $C_{\psi}$ on $H^{\infty}(V)$ are in the same norm vicinity as soon as they are in the same hyperbolic vicinity.

Proof. Since $V$ is connected, the spectrum of $H^{\infty}(V)$ is connected, and Corollary 3.5 applies. In this case, $\phi(V)$ and $\psi(V)$ are in the same Gleason part, namely that of $V$, so the second alternative of the corollary cannot occur.

\section{Subalgebras of Finite COdimension}

We wish to consider the restrictions of homomorphisms from $A$ to $B$ to subalgebras $A_{s}$ of $A$ of finite (linear) codimension. By Ga2, any such subalgebra can be embedded in a descending chain of subalgebras, $A=A_{0} \supset A_{1} \supset A_{2} \supset \cdots \supset$ $A_{m}=A_{s}$, where each $A_{k}$ has codimension 1 in $A_{k-1}$. Thus we need analyze only the case of a subalgebra $A_{s}$ of codimension 1 in $A$.

So we assume that $A_{s}$ is a subalgebra of $A$ of codimension 1. According to Ga2, there are two cases that can occur: either $A_{s}$ is obtained from $A$ by identifying two points of $M_{A}$, or $A_{s}$ is the null space of a point derivation at some point of $M_{A}$. In the former case, the spectrum $M_{A_{s}}$ is obtained from $M_{A}$ by identifying the two points identified by $A$. In the latter case, $M_{A_{s}}$ coincides with $M_{A}$. For $x \in M_{A}$, we denote by $\bar{x}$ the corresponding point of $M_{A_{s}}$, that is, the restriction of $x$ to $A_{s}$. For a subset $S$ of $M_{A}$, we denote by $\bar{S}=\{\bar{x}: x \in S\}$ the corresponding quotient subset of $M_{A_{s}}$.

We wish to relate the pseudohyperbolic metrics on $M_{A}$ and $M_{A_{s}}$. We denote by $\rho_{s}$ the pseudohyperbolic metric $\rho_{A_{s}}$ on the spectrum $M_{A_{s}}$ of $A_{s}$. Since $A_{s} \subset A$, we have

$$
\rho_{s}(\bar{x}, \bar{y}) \leq \rho_{A}(x, y), \quad x, y \in M_{A}
$$


We need estimates in the other direction. To compare $\rho_{s}$ and $\rho_{A}$, we divide the discussion into several cases.

Case 1. Suppose $A_{s}$ consists of the functions $f \in A$ such that $f\left(x_{0}\right)=f\left(x_{1}\right)$, where $x_{0}$ and $x_{1}$ belong to distinct Gleason parts $P_{0}$ and $P_{1}$ of $M_{A}$. Thus $\overline{x_{0}}=\overline{x_{1}}$. Let $x \in M_{A} \backslash\left(P_{0} \cup P_{1}\right)$, and let $y \in M_{A}$. We can find $f \in A$ such that $\|f\|<$ $1, f\left(x_{0}\right)=f\left(x_{1}\right)=f(y)=0$, and $f(x) \approx \rho_{A}(x, y)$. Since $f \in A_{s}$, we obtain $\rho_{s}(\bar{x}, \bar{y}) \geq \rho_{A}(x, y)$. Thus

$$
\rho_{s}(\bar{x}, \bar{y})=\rho_{A}(x, y)
$$

for $x, y \in M_{A}$, providing $x \notin P_{0} \cup P_{1}$. A similar argument shows that the identity (6) holds if both $x$ and $y$ belong to $P_{0}$, or if they both belong to $P_{1}$.

Suppose $x \in P_{0}$ and $y \in P_{1}$. Choose $f_{0} \in A$ such that $\left\|f_{0}\right\|<1, f_{0}\left(x_{0}\right)=0$, and $f_{0}(x) \approx \rho_{A}\left(x_{0}, x\right)$. Since $y$ is in a different part from $x$, we can arrange that $f_{0}(y) \approx 1$. Choose $f_{1} \in A$ such that $\left\|f_{1}\right\|<1, f_{1}\left(x_{1}\right)=0, f_{1}(y) \approx$ $-\rho_{A}\left(x_{1}, y\right)$, and $f_{1}(x) \approx 1$. Then $f=f_{0} f_{1} \in A_{s},\|f\|<1, f(x) \approx \rho_{A}\left(x_{0}, x\right)$, and $f(y) \approx-\rho_{A}\left(x_{1}, y\right)$. The pseudohyperbolic distance from $f(x)$ to $f(y)$ in $\mathbb{D}$ is then close to $\left(\rho_{A}\left(x_{0}, x\right)+\rho_{A}\left(x_{1}, y\right)\right) /\left(1+\rho_{A}\left(x_{0}, x\right) \rho_{A}\left(x_{1}, y\right)\right)$, and we obtain $\rho_{s}(\bar{x}, \bar{y}) \geq\left(\rho_{A}\left(x_{0}, x\right)+\rho_{A}\left(x_{1}, y\right)\right) /\left(1+\rho_{A}\left(x_{0}, x\right) \rho_{A}\left(x_{1}, y\right)\right)$. Since $x_{0}$ and $x$ are in the same Gleason part, $\rho_{A}\left(x_{0}, x\right)=\rho_{s}\left(\overline{x_{0}}, \bar{x}\right)$, and also $\rho_{A}\left(x_{1}, y\right)=\rho_{s}\left(\overline{x_{1}}, \bar{y}\right)$. Thus from König's inequality (3), applied to $\rho_{s}$, we obtain equality, that is,

$$
\rho_{s}(\bar{x}, \bar{y})=\frac{\rho_{A}\left(x_{0}, x\right)+\rho_{A}\left(x_{1}, y\right)}{1+\rho_{A}\left(x_{0}, x\right) \rho_{A}\left(x_{1}, y\right)} \quad x \in P_{0}, y \in P_{1} .
$$

There is a similar identity if $x \in P_{1}$ and $y \in P_{0}$, and in every other case (6) holds. This completely determines the pseudohyperbolic metric $\rho_{s}$ in terms of $\rho_{A}$. In particular, we see that the Gleason parts of $A_{s}$ are the same as the Gleason parts of $A$, except that the two Gleason parts $P_{0}$ and $P_{1}$ of $A$ are joined at $\overline{x_{0}}=\overline{x_{1}}$ to form a single part of $A_{s}$. Note that the identities connecting $\rho_{A}$ and $\rho_{s}$ show that a subset of $M_{A}$ is hyperbolically bounded if and only if the corresponding quotient subset of $M_{A_{s}}$ is hyperbolically bounded.

Case 2. Suppose $A_{s}$ consists of the functions $f \in A$ such that $f\left(x_{0}\right)=f\left(x_{1}\right)$, where $x_{0}$ and $x_{1}$ belong to the same Gleason part $P$ of $M_{A}$. As before, the identity (6) holds unless $x$ and $y$ both belong to $P$. Thus the Gleason parts of $A_{s}$ are the same as those of $A$.

Suppose $x \in P$. Choose $f \in A_{s}$ such that $\|f\|<1, f\left(\overline{x_{0}}\right)=0$, and $f(\bar{x}) \approx$ $\rho_{s}\left(\overline{x_{0}}, \bar{x}\right)$. Since $f\left(x_{0}\right)=0,|f(x)| \leq \rho_{A}\left(x_{0}, x\right)$, and since $f\left(x_{1}\right)=0,|f(x)| \leq$ $\rho_{A}\left(x_{1}, x\right)$. Hence

$$
\rho_{s}\left(\overline{x_{0}}, \bar{x}\right) \leq \min \left(\rho_{A}\left(x_{0}, x\right), \rho_{A}\left(x_{1}, x\right)\right), \quad x \in P .
$$

For a lower bound, choose $f_{0} \in A$ such that $\left\|f_{0}\right\|<1, f_{0}\left(x_{0}\right)=0$, and $f_{0}(x) \approx$ $\rho_{A}\left(x_{0}, x\right)$, and similarly choose $f_{1} \in A$ such that $\left\|f_{1}\right\|<1, f_{1}\left(x_{1}\right)=0$, and $f_{1}(x) \approx \rho_{A}\left(x_{1}, x\right)$. Then $f=f_{0} f_{1} \in A_{s}, f\left(\overline{x_{0}}\right)=0$, and $f(\bar{x}) \approx \rho_{A}\left(x_{0}, x\right) \rho_{A}\left(x_{1}, x\right)$. It follows that

$$
\min \left(\rho_{A}\left(x_{0}, x\right)^{2}, \rho_{A}\left(x_{1}, x\right)^{2}\right) \leq \rho_{A}\left(x_{0}, x\right) \rho_{A}\left(x_{1}, x\right) \leq \rho_{s}\left(\overline{x_{0}}, \bar{x}\right), \quad x \in P .
$$

As before, these estimates show that a subset of $M_{A}$ is hyperbolically bounded if and only if the corresponding quotient subset of $M_{A_{s}}$ is hyperbolically bounded.

Continuing with $f_{0}$ and $f_{1}$, suppose that $y \in P$, and choose $g \in A$ such that $\|g\|<1, g(y)=0$ and $g(x) \approx \rho_{A}(x, y)$. Then $h=g f_{1} f_{2} \in A_{s},\|h\|<1, h(y)=0$, 
and $h(x) \approx \rho_{A}\left(x_{0}, x\right) \rho_{A}\left(x_{1}, x\right) \rho_{A}(x, y)$. Consequently

$$
\rho_{A}\left(x_{0}, x\right) \rho_{A}\left(x_{1}, x\right) \rho_{A}(x, y) \leq \rho_{s}(\bar{x}, \bar{y}), \quad x, y \in P .
$$

This shows that as $x$ tends to $\infty$ in the part $P$, the metrics $\rho_{A}(x, y)$ and $\rho_{s}(\bar{x}, \bar{y})$ are close to each other.

Case 3. Suppose that $A_{s}$ is the null space of a point derivation at some point $x_{0} \in M_{A}$, and let $P$ denote the Gleason part containing $x_{0}$. Then $A_{s}$ includes all products of the form $f g$, where $f, g \in A$ satisfy $f\left(x_{0}\right)=g\left(x_{0}\right)=0$. Case 3 may be regarded as a limiting case of Case 2 as $x_{1} \rightarrow x_{0}$. If we proceed in analogy with Case 2, with $x_{0}=x_{1}$, we see that the Gleason parts of $A_{s}$ are the same as those of $A$, and the identity (6) holds unless both $x$ and $y$ belong to $P$. The analogs of the estimates (9) and (10), obtained in the same way, are

$$
\begin{gathered}
\rho_{A}\left(x_{0}, x\right)^{2} \leq \rho_{s}\left(\overline{x_{0}}, \bar{x}\right), \quad x \in P, \\
\rho_{A}\left(x_{0}, x\right)^{2} \rho_{A}(x, y) \leq \rho_{s}(\bar{x}, \bar{y}), \quad x, y \in P .
\end{gathered}
$$

Again the estimates show that a subset of $M_{A}$ is hyperbolically bounded if and only if the corresponding quotient subset of $M_{A_{s}}$ is hyperbolically bounded, and the metrics $\rho_{A}(x, y)$ and $\rho_{s}(\bar{x}, \bar{y})$ are close to each other as $x$ tends to $\infty$ in the part $P$.

We summarize some of these results in the following theorem.

Theorem 4.1. Let $A_{s}$ be a unital subalgebra of finite codimension in the uniform algebra $A$. Then the spectrum $M_{A_{s}}$ is obtained from $M_{A}$ by identifying a finite number of pairs of points. A subset $E$ of $M_{A}$ is hyperbolically bounded in $M_{A}$ if and only if the corresponding quotient subset of $M_{A_{s}}$ is hyperbolically bounded in $M_{A_{s}}$.

Proof. This follows from the corresponding results in each of the three cases treated above and the reduction of the general case to the case of codimension 1.

Suppose now that $C_{\phi}$ and $C_{\psi}$ are unital homomorphisms from the uniform algebra $A$ to a uniform algebra $B$, corresponding to maps $\phi, \psi: M_{B} \rightarrow M_{A}$. Let $A_{s}$ be a unital subalgebra of $A$ of finite codimension. The restrictions of $C_{\phi}$ and $C_{\psi}$ to $A_{s}$ are unital homomorphisms from $A_{s}$ to $B$, corresponding to maps $\bar{\phi}, \bar{\psi}: M_{B} \rightarrow M_{A_{s}}$ obtained respectively by following $\phi$ and $\psi$ by the quotient map of $M_{A}$ onto $M_{A_{s}}$, which simply identifies the (finite number of) pairs of points in $M_{A}$ that are identified by $A_{s}$.

Theorem 4.2. Let $A$ and $B$ be uniform algebras, and let $A_{s}$ be a unital subalgebra of $A$ of finite (linear) codimension. Let $C_{\phi}$ and $C_{\psi}$ be composition operators from $A$ to $B$, and let $C_{\bar{\phi}}$ and $C_{\bar{\psi}}$ be their restrictions to $A_{s}$. Then $C_{\phi}$ and $C_{\psi}$ belong to the same hyperbolic vicinity if and only if $C_{\bar{\phi}}$ and $C_{\bar{\psi}}$ belong to the same hyperbolic vicinity.

Proof. The estimate (4) and Theorem 4.1 show that if $C_{\phi}$ and $C_{\psi}$ are in the same hyperbolic vicinity, then so are $C_{\bar{\phi}}$ and $C_{\bar{\psi}}$. We must show the converse. As before we assume that $A_{s}$ is a subalgebra of $A$ of codimension 1 .

Assume that $C_{\bar{\phi}}$ and $C_{\bar{\psi}}$ are in the same hyperbolic vicinity. Let $E$ denote the set of $y \in M_{B}$ such that $\overline{\phi(y)}$ and $\overline{\psi(y)}$ belong to the same Gleason part of $M_{A_{s}}$. Then $\overline{\phi\left(M_{B} \backslash E\right)}$ and $\overline{\psi\left(M_{B} \backslash E\right)}$ are hyperbolically bounded in $M_{A_{s}}$, so $\phi\left(M_{B} \backslash E\right)$ and $\psi\left(M_{B} \backslash E\right)$ are hyperbolically bounded in $M_{A}$. Now $\rho_{s}(\overline{\phi(y)}, \overline{\psi(y)})=\rho_{A}(\phi(y), \psi(y))$ 
whenever $y \in E$, and $\phi(y), \psi(y)$ are in some part other than $P_{0}$ or $P_{1}$ (in Case 1) or $P$ (in Cases 2 or 3). The estimates (10) in Case 2 and (12) in Case 3 show that $\rho_{A}(\phi(y), \psi(y)) \leq r^{\prime}$ for some $r^{\prime}<1$ and all $y \in E$ such that $\phi(y), \psi(y) \in P$, so that $C_{\phi}$ and $C_{\psi}$ are in the same hyperbolic vicinity in Cases 2 and 3.

So we assume we are in Case 1 , with the previous notation. In this case, if $\phi(y), \psi(y)$ both belong to the same Gleason part of $M_{A}$, then $\rho_{s}(\overline{\phi(y)}, \overline{\psi(y)})=$ $\rho_{A}(\phi(y), \psi(y))$. To complete the proof, it suffices to show that if $E_{0}$ is the set of $y \in E$ such that $\phi(y) \in P_{0}$ and $\psi(y) \in P_{1}$, and $E_{1}$ is the set of $y \in E$ such that $\phi(y) \in P_{1}$ and $\psi(y) \in P_{0}$, then $\phi\left(E_{0}\right), \phi\left(E_{1}\right), \psi\left(E_{0}\right), \psi\left(E_{1}\right)$ are hyperbolically bounded in $M_{A}$. The identity (7) and the bound $\rho_{s}(\overline{\phi(y)}, \overline{\psi(y)}) \leq r$ show that $\rho_{A}\left(\phi(y), x_{0}\right) \leq r^{\prime}<1$ for $y \in E_{0}$, so that $\phi\left(E_{0}\right)$ and $\psi\left(E_{0}\right)$ are hyperbolically bounded. Similarly, $\phi\left(E_{1}\right)$ and $\psi\left(E_{1}\right)$ are hyperbolically bounded.

Corollary 4.3. Two composition operators $C_{\phi}$ and $C_{\psi}$ from $A$ to $B$ belong to the same hyperbolic vicinity if and only if there is a unital subalgebra $A_{s}$ of $A$ of finite (linear) codimension such that the restrictions of $C_{\phi}$ and $C_{\psi}$ to $A_{s}$ belong to the same norm vicinity, that is, $\left\|C_{\bar{\phi}}-C_{\bar{\psi}}\right\|<2$.

Proof. If $\left\|C_{\bar{\phi}}-C_{\bar{\psi}}\right\|<2$, the operators $C_{\bar{\phi}}$ and $C_{\bar{\psi}}$ are in the same hyperbolic vicinity with respect to $A_{s}$, hence by Theorem $4.2, C_{\phi}$ and $C_{\psi}$ are in the same hyperbolic vicinity. For the converse, suppose $C_{\phi}$ and $C_{\psi}$ are in the same hyperbolic vicinity. Take clopen subsets $E_{\alpha}$ in $M_{B}$ as in Theorem 3.4 so that $\phi\left(E_{\alpha}\right)$ and $\psi\left(E_{\alpha}\right)$ are hyperbolically bounded sets in different Gleason parts, say $P_{\alpha}$ and $Q_{\alpha}$. For each $\alpha$, select an arbitrary pair of points $x_{\alpha} \in P_{\alpha}$ and $x_{\alpha}^{\prime} \in Q_{\alpha}$, and let $A_{s}$ be the collection of functions $f \in A$ such that $f\left(x_{\alpha}\right)=f\left(x_{\alpha}^{\prime}\right)$ for all $\alpha$. Then $A_{s}$ is a subalgebra of $A$ of finite codimension. By Theorem 4.2, the restriction composition operators $C_{\bar{\phi}}$ and $C_{\bar{\psi}}$ are in the same hyperbolic vicinity. Since $\overline{\phi(y)}$ and $\overline{\psi(y)}$ belong to the same Gleason part of $M_{A_{s}}$ for all $y \in M_{B}$, Theorem 3.4 shows that $C_{\bar{\phi}}$ and $C_{\bar{\psi}}$ belong to the same norm vicinity.

\section{HyPERBOLIC VICINITIES AND ESSENTIAL OPERATOR NORMS}

We are now ready to apply Lemma 2.1 to see that hyperbolic vicinities are open in the essential operator norm.

Theorem 5.1. If the composition operators $C_{\phi}$ and $C_{\psi}$ from $A$ to $B$ satisfy $\left\|C_{\phi}-C_{\psi}\right\|_{e}<1$, then $C_{\phi}$ and $C_{\psi}$ belong to the same hyperbolic vicinity.

Proof. Let $E$ be the set of $y \in M_{B}$ such that $\phi(y)$ and $\psi(y)$ belong to the same Gleason part of $A$. Suppose $\left\{y_{j}\right\}$ is a sequence in $E$ such that $\rho_{A}\left(\phi\left(y_{j}\right), \psi\left(y_{j}\right)\right) \rightarrow 1$. If both $\left\{\phi\left(y_{j}\right)\right\}$ and $\left\{\psi\left(y_{j}\right)\right\}$ are hyperbolically bounded, then König's inequality (3) yields $\rho_{A}\left(\phi\left(y_{j}\right), \psi\left(y_{j}\right)\right) \leq s<1$, contradicting our assumption. Thus we can assume that either $\left\{\phi\left(y_{j}\right)\right\}$ or $\left\{\psi\left(y_{j}\right)\right\}$ is not hyperbolically bounded. However, we now deduce from Lemma 2.1 that $\left\|C_{\phi}-C_{\psi}\right\|_{e} \geq 1$, in contradiction to our hypothesis. We conclude that there is $r<1$ such that $\rho_{A}(\phi(y), \psi(y)) \leq r$ for all $y \in E$.

Now $\rho_{A}(\phi(y), \psi(y))=1$ for all $y \in M_{B} \backslash E$. Our hypothesis and Lemma 2.1 then show that $\left\{\phi\left(y_{j}\right)\right\}$ is hyperbolically bounded for any sequence in $M_{B} \backslash E$, and consequently $\phi\left(M_{B} \backslash E\right)$ is hyperbolically bounded, as is $\psi\left(M_{B} \backslash E\right)$.

Corollary 5.2. Hyperbolic vicinities of composition operators are open in the essential operator norm. 
Thus hyperbolic vicinities of composition operators form a partition of the set of composition operators from $A$ to $B$ into disjoint subsets that are clopen with respect to the essential operator norm.

Combining Corollary 5.2 and Corollary 3.5, we obtain the following.

Corollary 5.3. If $M_{B}$ is connected, and if $C_{\phi}$ is a composition operator from $A$ to $B$ such that $\phi\left(M_{B}\right)$ is not hyperbolically bounded, then the norm vicinity of $C_{\phi}$ is open with respect to the essential operator norm.

\section{HyPERBOLIC VICINITY OF THE IDENTITY OPERATOR}

In [HIZ, T. Hosokava, K. Izuchi, and D. Zheng characterize the composition operators on $H^{\infty}(\mathbb{D})$ arising from analytic self-maps of $\mathbb{D}$ that are isolated with respect to the uniform operator norm. Further, they show that such operators are isolated with respect to the essential operator norm. In particular, the identity operator on $H^{\infty}(\mathbb{D})$ is isolated. It turns out that this result is quite general, as the following theorem shows.

Theorem 6.1. A composition operator $C_{\psi}$ on a uniform algebra $A$ belongs to the same hyperbolic vicinity as the identity operator $I$ on $A$ if and only if $\psi(x)=x$ for all $x \in M_{A}$ with the possible exception of finitely many isolated points of $M_{A}$. In particular, if $M_{A}$ has no isolated points, then the identity operator on $A$ is isolated with respect to the essential operator norm in the set of composition operators on A.

Proof. Note that $I=C_{\phi}$ for $\phi(x)=x, x \in M_{A}$. We consider the decomposition of $M_{A}$ as a disjoint union of $E$ and the (finitely many) sets $E_{\alpha}$ given by Theorem 3.4. Since $E_{\alpha}$ is clopen, it has a strong boundary point $x_{\alpha}$. Since $\phi\left(E_{\alpha}\right)$ is contained in one Gleason part, and strong boundary points are one-point parts, we have $\phi(x)=x_{\alpha}$ for $x \in E_{\alpha}$, and $E_{\alpha}$ coincides with the singleton $\left\{x_{\alpha}\right\}$. Thus all the $E_{\alpha}$ 's are singletons, which are isolated points of $M_{A}$.

On $E$ we have $\rho_{A}(x, \psi(x)) \leq r<1$. If $x \in E$ is a strong boundary point of $A$, then it comprises a one-point part, and consequently $\psi(x)=x$. Since the strong boundary points are dense in the Shilov boundary, this identity persists for all $x$ in the Shilov boundary of the restriction algebra $\left.A\right|_{E}$ of $A$ to $E$. Consequently $C_{\psi} f=f$ on the Shilov boundary of the restriction algebra. It follows that $C_{\psi} f=f$ on the spectrum $E$ of the restriction algebra. Thus $\psi(x)=x$ for $x \in E$.

It was conjectured in AGL that the identity operator on $H^{\infty}\left(B_{X}\right)$ is isolated in the set of composition operators endowed with the uniform operator norm. The conjecture was settled affirmatively in $[\mathrm{CHM}]$. Actually, the identity operator on any uniform algebra $A$ is isolated in the set of composition operators on $A$ with respect to the uniform operator norm. In fact, the identity operator forms by itself a norm vicinity. To see this, suppose that $C_{\psi}$ belongs to the same norm vicinity as the identity operator. Then $\rho(x, \psi(x)) \leq r<1$ for all $x \in M_{A}$, and we can take $E=M_{A}$ in the preceding proof. We conclude that $\psi(x)=x$ for all $x \in M_{A}$, and $C_{\psi}=I$.

A theorem analogous to Theorem 6.1 can be formulated for composition operators from $A$ to $B$.

Theorem 6.2. Let $A$ and $B$ be uniform algebras such that $M_{B}$ is connected. Let $C_{\phi}$ be a composition operator from $A$ to $B$. Suppose that $\phi$ maps strong boundary 
points in $M_{B}$ to strong boundary points in $M_{A}$, and that $\phi$ is not constant. Then $\left\{C_{\phi}\right\}$ comprises by itself a hyperbolic vicinity. In particular, $C_{\phi}$ is isolated with respect to the essential operator norm in the set of composition operators from $A$ to $B$.

Proof. If $C_{\psi}$ is in the same hyperbolic vicinity as $C_{\phi}$, then in this case the sets $E_{\alpha}$ in Theorem 3.4 must be empty, and as before we obtain $\psi(y)=\phi(y)$ for strong boundary points, hence for all $y \in M_{B}$.

\section{REFERENCES}

[AGL] R. Aron, P. Galindo, and M. Lindström, Connected components in the space of composition operators on analytic functions of many variables, Int. Eq. Op. Theory 45 (2003), 1-14. MR1952340 (2003m:47041)

$[\mathrm{CHM}]$ C. H. Chu, R. V. Hügli, and M. Mackey, The identity is an isolated composition operator in $H^{\infty}(B)$, Proc. Amer. Math. Soc. 132 (2004), no. 11, 3305-3308 (electronic). MR2073306 (2005b:46091)

[Da] A. Davie, Linear extension operators for spaces and algebras of functions, Amer. J. Math. 94 (1972), 156-172. MR0300093 (45:9141)

[GGL] P. Galindo, T. W. Gamelin, and M. Lindström, Composition operators on uniform algebras and the pseudohyperbolic metric, J. Korean Math. Soc. 41 (2004), 1-20. MR2048697 (2004m:47047)

[GL] P. Galindo and M. Lindström, Factorization of homomorphisms through $H^{\infty}(D)$, J. of Math. Anal. and Appl. 280 (2003), 375-386. MR1977918 (2004g:46067)

[Ga1] T. Gamelin, Uniform Algebras, second edition, AMS Chelsea Publishing, 1984. MR0410387(53:14137)

[Ga2] T. Gamelin, Embedding Riemann surfaces in maximal ideal spaces, J. Functional Anal. 2 (1968), 123-146. MR0223894 (36:6941)

[Ga3] T. Gamelin, Uniform algebras on plane sets, in Approximation Theory, edited by G.G. Lorentz, Academic Press, 1973, 101-149. MR0338784 (49:3548)

[Ga4] T. Gamelin, Homomorphisms of uniform algebras, in Recent Progress in Functional Analysis, edited by Bierstedt et al., Elsevier/North-Holland, 2001, 95-105. MR 1861749 (2002h:46084)

[GM] P. Gorkin and R. Mortini, Norms and essential norms of linear combinations of endomorphisms, Trans. Amer. Math. Soc. 358 (2006), 553-571. MR2177030

[HIZ] T. Hosokava, K. Izuchi, and D. Zheng, Isolated points and essential components of composition operators on $H^{\infty}$ functions, Proc. Amer. Math. Soc. 130 (2002), 1765-1773. MR1887024 (2003d:47033)

[Ka] H. Kamowitz, Compact endomorphisms of Banach algebras, Pac. J. Math. 89 (1980), 313-325. MR0599123 (82c:46063)

[Kl] U. Klein, Kompakte multiplikative Operatoren auf uniformen Algebren, Mitt. Math. Sem. Giessen No. 232, 1997, iv + 120 pp. MR1479364(99b:47043)

[Ko] H. König, Zur abstrakten theorie der analytischen funktionen II, Math. Ann. 163 (1966), 9-17. MR0190792(32:8202)

[Zh] L. Zheng, The essential norms and spectra of composition operators on $H^{\infty}$, Pacific J. Math. 203 (2002), 503-510. MR1897912 (2003e:47052)

Departamento de Análisis Matemático, Universidad de Valencia, 46.100, Burjasot, Valencia, Spain

E-mail address: galindo@uv.es

Department of Mathematics, University of California los Angeles, Los Angeles, CALifornia 90095-1555

E-mail address: twg@math.ucla.edu

Department of Mathematics, Abo Akademi University, Fin-20500 Abo, Finland

E-mail address: mlindstr@abo.fi 